\title{
Intrahepatic branching of the portal vein in the Eurasian otter (Lutra lutra) and American mink (Neovison vison)
}

\author{
Nedžad HADŽIOMEROVIĆ ${ }^{1, a, ®}$, Rizah AVDIĆc ${ }^{1, b}$, Faruk TANDIR $^{1, \mathrm{c}}$, Ermin ŠALJĆ $^{2, \mathrm{~d}}$, \\ Pamela BEJDIĆ ${ }^{1, e}$
}

\author{
${ }^{1}$ University of Sarajevo, Veterinary Faculty, Department of Basic Sciences of Veterinary Medicine, Sarajevo, Bosnia and \\ Herzegovina; ${ }^{2}$ University of Sarajevo, Veterinary Faculty, Department of Clinical Sciences of Veterinary Medicine, Sarajevo, Bosnia \\ and Herzegovina \\ a ORCID: 0000-0002-3155-1011; ' ORCID: 0000-0002-6874-444X; ' ORCID: 0000-0001-9184-592X; \\ dORCID: 0000-0001-5399-4916; ' ORCID: 0000-0002-4938-6333 \\ Corresponding author: nedzad.hadziomerovic@vfs.unsa.ba \\ Received date: 20.11.2020 - Accepted date: 21.08.2021
}

\begin{abstract}
The study aimed to evaluate the comparative anatomy of the liver and intrahepatic branching of the portal vein of the Eurasian otter (Lutra lutra) and the American mink (Neovison vison). Due to their highly valuable fur, minks have expanded their range to many parts of Europe and become available for many biomedical studies. In this study, ten adult minks and five otters were used. The intrahepatic branching of the portal vein was studied by the combined injection and dissection technique. The macroscopic anatomy of the liver revealed that both species have six-lobed livers, although differences in shape, size and some additional fissures were documented. The portal vein, upon entering the liver, divides into the right and left branches. The branching pattern in otters had an additional branch at this level with a caudate process branch. The right branch of the portal vein ramified in the right lateral lobe and the caudate process in the mink livers, while the right branch in the otter livers only distributed functional blood to the right lateral lobe. The larger left portal branch, with its transverse and umbilical parts, ramified in the left liver portion, along with the quadrate, right medial lobe and papillary process.
\end{abstract}

Keywords: Lutra lutra, Neovison vison, portal vein, vascular casts.

\section{Introduction}

The Eurasian otter and American mink both belong to the large carnivore family of Mustelidae, which includes similar animals, such as the weasel, ferret, marten, badger and wolverine. They vary in size with similar long slender bodies, short legs and a long tail (13). The fully aquatic otters are mostly found in the wild and have developed adaptations to marine life, while the semiaquatic mink has expanded its range due to the human interest in the fur industry, which has become very intense over the course of the last twenty years. Minks have become available for many studies as dissection models, in experimental surgery, as well as a potential biomedicine model (11). Some research has used mink as a model in toxicology (2), sinus inflammation (12), investigation of Aleutian disease (9), etc. The significance of the mink liver as a potential model for surgical resection, physiological, clinical and pathological investigation is great, due to its lobation similar to domestic canid species, and larger body in comparison with traditional laboratory animals. Smith and Schenk (21) gave a basic overview of the morphology, topography and lobation of the liver in the mink, while a detailed study of the intrahepatic ramification of hepatic veins and biliary ducts has been well documented (6). Basic insights into the otter liver revealed that the otter has six or seven hepatic lobes (1, 13). The intrahepatic branching of the portal vein was described in dogs $(3,10,24)$ and cats $(8)$, but also in other domestic animals such as sheep (7), rabbits (8), horse (23), buffalo (18), camels (22), as well as in humans (5). The aim of this study was to investigate the comparative macroscopic anatomy of mink and otter livers, and the intrahepatic branching of the portal vein. Potential use in the field of surgery requires a good understanding of the anatomy and vasculature of an organ. The available literature found about this specific field is quite unclear and poor.

\section{Materials and Methods}

Ten adult minks and five otters were studied. Minks of both sexes were obtained from a fur farm, where the animals were euthanized and the carcasses remained 
undamaged. The animals were treated in accordance with the relevant legislation, Official Journal B\&H No. 34/02. Of November 22 (16). Otters were obtained from the Department of Pathology, Veterinary Faculty, University of Sarajevo, where they were brought for the autopsy. The dissection was performed and connective tissue was removed for the macro morphological study of the livers. The blood vessels were identified on the visceral surface, and the portal vein was ligated and rinsed with $0.9 \%$ saline solution. The casts were obtained using a combined injection and dissection technique (17). The acrylic monomer (Interacryl cold - Interdent, Slovenia) powder and solvent forms were mixed in 1:2 proportions. The mass was colored with a polyurethane green pigment (Biodur, AC54) for the portal vein and injected manually. Upon injection, the livers were left at room temperature for 24 hours to complete the polymerization of the resin and then placed in a 5\% formaldehyde solution for 10 days. The dissection was performed at the visceral surface of the liver until intrahepatic branches of the portal vein became visible. The terminology used in the study is in accordance with current anatomical terminology (14).

\section{Results}

The liver is a light brown gland with an average weight for minks of $51.75 \pm 2.98 \mathrm{~g}$ and in otters of 151.6 $\pm 6.65 \mathrm{~g}$. The diaphragmatic surfaces of both livers are smooth and convex, while the visceral surfaces are marked by the hepatic porta (porta hepatis) and the gall bladder (vesica fellea). The latter is located between the quadrate and right medial lobe of the liver, slightly dorsally in the otter. At the hepatic porta, the bile ducts leave and the portal vein and hepatic artery enter the liver. Several fissures extend from the ventral border and divide both livers into six lobes: left lateral lobe (LLL), left medial lobe (LML), quadrate lobe (QL), right medial lobe (RML), right lateral lobe (RLL) and caudate lobe (CL), subdivided into caudate (CP) and papillary process (PP) (Figure 1). Differences in the shape and size of the lobes were noticed. The right lateral lobe in the otter is subdivided by an additional fissure into two parts which resemble two separate lobes. Also, both processes of the caudate lobe are significantly larger than those in the mink liver. In most cases, the papillary process in the otter is twice as long and located over the hepatic porta. The caudate process forms a deep impression on the right kidney.

The portal vein enters the liver at the porta and divides into right (ramus dexter) and left (ramus sinister) branches in the liver of the mink (Figure 2). The branching pattern of the portal vein in otters is different because the caudate process branch ramifies from the portal vein at the same level as where the two major branches originate. The strong caudate process branch in otters, with 3-5 secondary branches, is directed dorsolaterally and distributes functional blood for that hepatic lobe. As for mink, the same branch originates from the right portal branch and has two smaller branches, due to the smaller size of the caudate process. Two or three branches from the ramus dexter are directed ventrolaterally toward the right lateral lobe - right lateral lobe branches (Figure 2). The vascular network of the right lateral lobe in otters is larger, because this lobe is separated into two parts by a deep fissure.

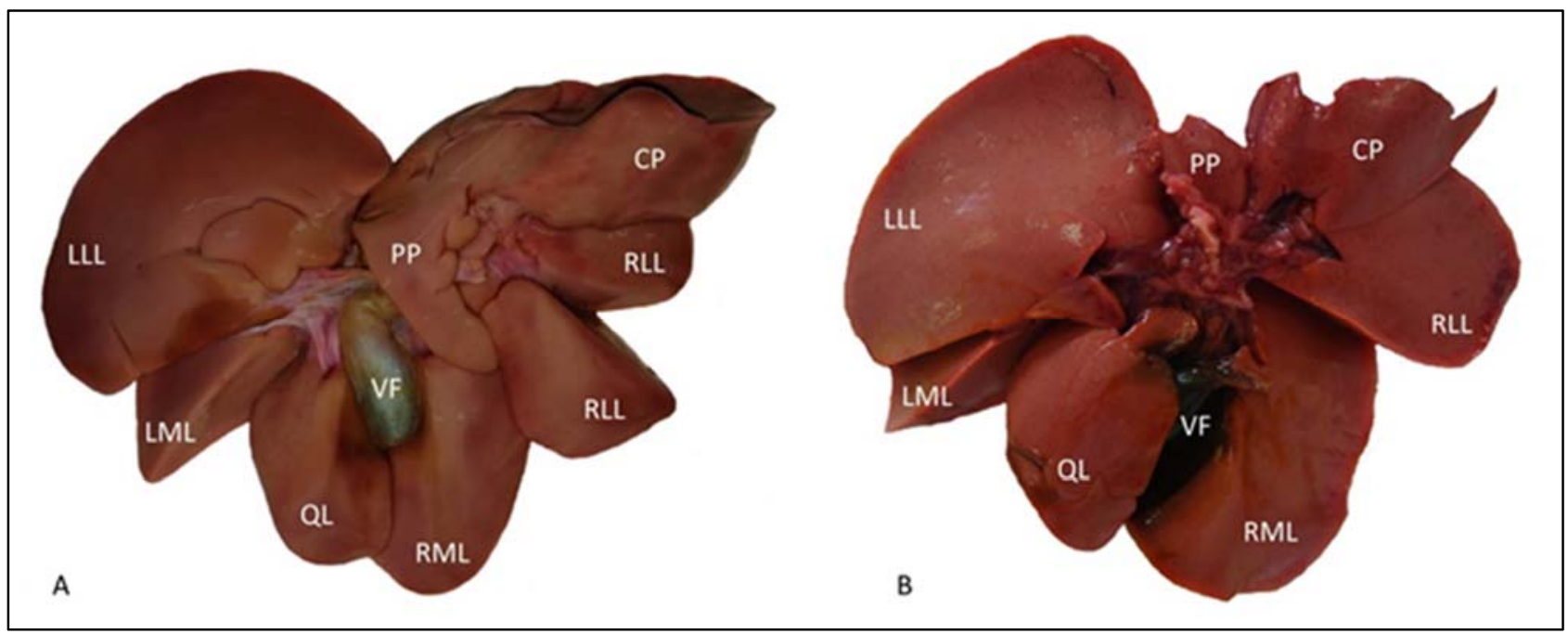

Figure 1. Visceral aspect of the liver. A - otter, B - mink.

LLL - left lateral lobe; LML - left medial lobe; QL - quadrate lobe; RML - right medial lobe; RLL - right lateral lobe; CP - caudate process; PP - papillary process; VF - gall bladder. 


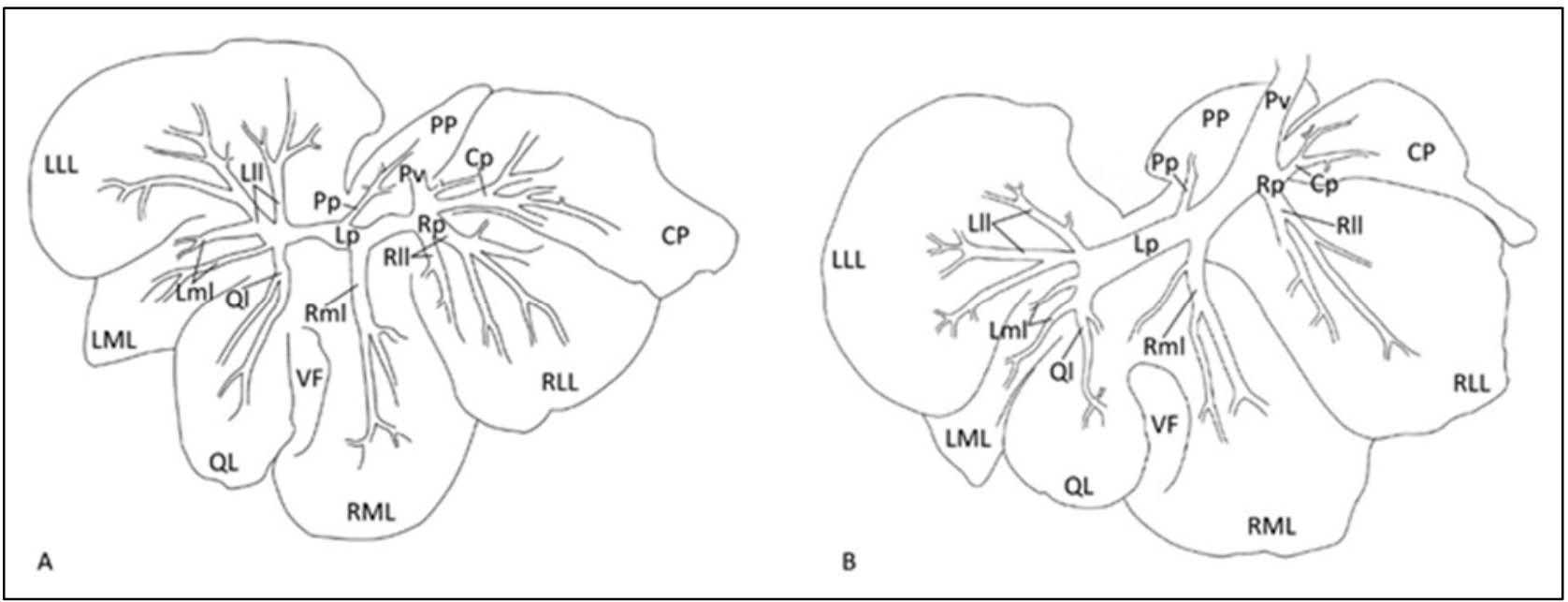

Figure 2. The portal vein ramification. A - otter, B - mink.

LLL - left lateral lobe; LML - left medial lobe; QL - quadrate lobe; RML - right medial lobe; RLL - right lateral lobe; CP - caudate process; PP - papillary process; VF - gall bladder; Pv - portal vein; Rp - right portal branch; Lp - left portal branch; Cp - caudate process branch; Pp - papillary process branch; Rll - right lateral lobe branches; Rml - right medial lobe branches; Q1 - quadrate lobe branches; Lml - left medial lobe branches; Lll - left lateral lobe branches.

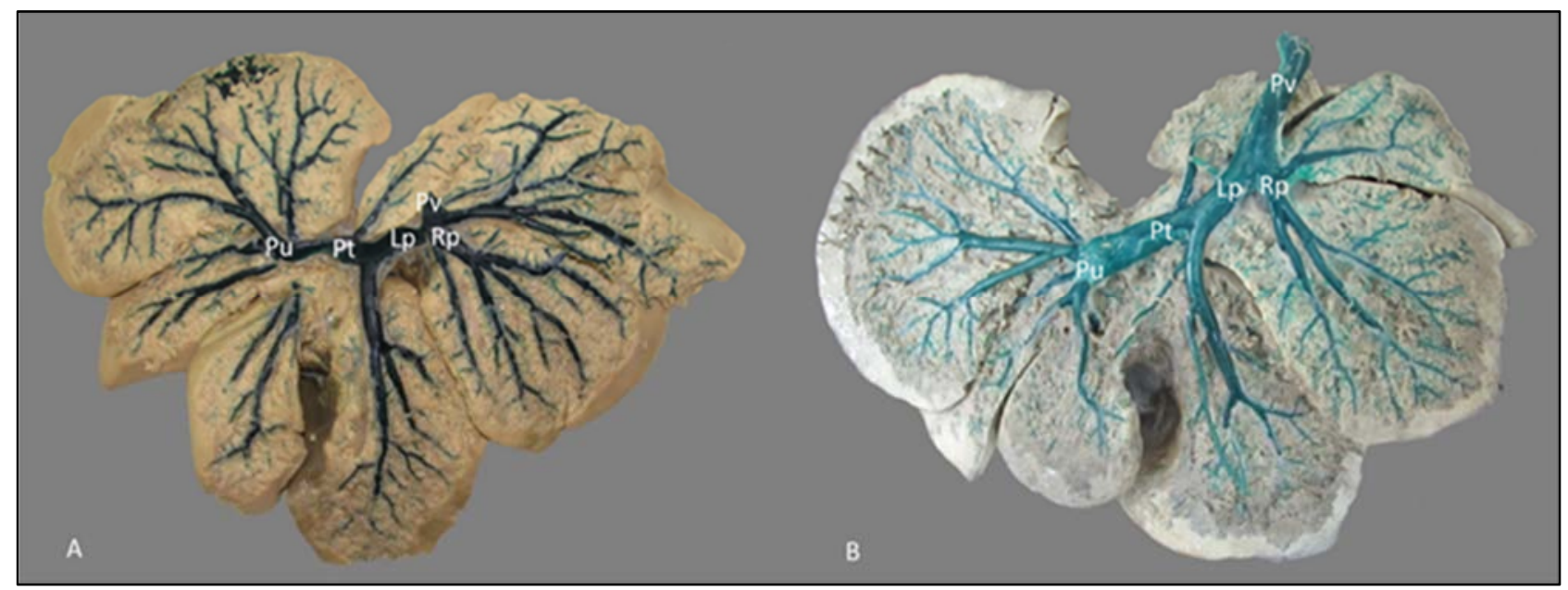

Figure 3. Corrosion cast of the portal vein. A - otter, B - mink.

$\mathrm{Pv}$ - portal vein; Rp - right portal branch; Lp - left portal branch; Pt - transverse part; $\mathrm{Pu}$ - umbilical part.

The left portal branch, in terms of size and direction, represents the continuation of the portal vein. The initial transverse part (pars transversa) is directed transversally and slightly ventrally, whilst the terminal umbilical part (pars umbilicalis) runs between the quadrate and left medial lobe in the form of an arc (Figure 3). This portal branch ramifies in the left liver portion, along with the quadrate, right medial lobe and papillary process. The first branch of the ramus sinister is directed ventrally and ramifies in the right medial lobe in both livers. The mink liver has an additional small branch directed towards the gall bladder from the right medial lobe branch. The single papillary process branch is directed dorsally from the transverse part. The final ramification of the left portal branch is at the level of the quadrate lobe, where the branches are directed towards the left lateral, left medial and quadrate lobes. The three branches are directed dorsolaterally and distributed in the left lateral lobe, two branches for the left medial lobe and two branches for the quadrate lobe. This branching pattern is similar in both the mink and the otter livers. The parietal surface of the liver exhibit terminal branches of the portal vessels. The position of the portal vein branches were visceral comparing to the branches of the hepatic veins which lies deeper and parietal.

\section{Discussion and Conclusion}

The liver, as the largest gland, has both exocrine and endocrine functions. The exocrine product, bile, is stored in the gall bladder, which is located between the quadrate 
and right medial lobes of the liver. The six lobes of the carnivore liver are divided with several fissures from the ventral border (3, 4). Basic insights into mink livers reveal typical carnivore lobation (six lobes), shape and structure (21). The available literature on otter livers revealed a different lobation pattern. According to Baitchman and Kollias (1), the otter liver is grossly divided into six lobes, while a recent study (13) described otter liver as sevenlobed. We found that the otter liver has six lobes because the additional fissure of the right lateral lobe did not separate the lobe completely. Also, in terms of vasculature, the branching pattern showed two main branches that distributed portal blood to the complete right lateral lobe. The portal vein with its associated blood vessels forms a hepatic portal system. It collects blood from abdominal organs, except the terminal rectum, and terminates with a capillary bed in the liver (19). A detailed description of the portal vein distribution in dogs was given by Kalt and Stump (10). The portal vein divides at the porta into smaller right and larger left branches. The right branch ramifies in the caudate process of the caudate lobe and the right lateral lobe of the liver. The larger left branch gives off 5-7 branches for the right medial, quadrate, left medial, left lateral and papillary process of the caudate lobe. Similar ramifications in dog were described by Uršič et al. (24), and Sleight and Thomford (20). Our findings were in accordance with previous investigations in dogs regarding the individual branches entering the hepatic lobes. However, Uršič et al. (24) reported cases where the left portal branch supplied the medial part of the right lateral lobe of the liver, which was contrary to our findings and a previous study in dogs by Kalt and Stump (10). Also, the papillary process vein consists of 2-3 larger branches (24), while the present study describes a single papillary process branch, in both mink and otter. Heath and House (8) reported three main branches of the portal vein in cats. The caudate branch supplies the caudate lobe, the right branch supplies the right part of the liver from the gall bladder, and the left branch supplies the left lateral, left medial and quadrate lobes. In rabbits, the first branch of the portal vein emerges dorsally and supplies the caudate lobe. Then the portal vein continues in a cranioventral direction and divides into right and left branches (8). The portal vein ramification in the liver of horses is different from other mammals, as it divides into six branches: the caudate, dorsal diaphragmatic, right caudodorsal, right intermediate, right common and left branches (23). The portal vein of buffalo divides at the porta into short right and long left branches. The right branch gives off several interlobular branches that ramify in the right lobe, both processes of the caudate lobe and the quadrate lobe. The left branch consists of a transverse and an umbilical part. The transverse part has several branches for the papillary process and the quadrate lobe, while the umbilical part gives off three branches that ramify in the left and quadrate lobes (18). The portal vein is connected through the hepatic capillary system and the hepatic veins to the caudal vena cava (15) but no interconnecting branches was found between these vessels at our macroanatomic vascular study. The present study revealed detailed insights into the livers of two species belonging to the Mustelidae family. The results show that both livers consist of six lobes, although differences in the shape, size and additional interlobar fissures were documented. Both species exhibit similar intrahepatic distribution of the portal vein, which will be valuable for the further use in the field of surgery, pathology etc.

\section{Financial Support}

This research received no grant from any funding agency/sector.

\section{Ethical Statement}

This study does not present any ethical concerns.

\section{Conflict of Interest}

The authors declared that there is no conflict of interest.

\section{References}

1. Baitchman E, Kollias GV (2000): Clinical anatomy of the North American river otter (Lontra canadensis). J Zoo Wildlife Med, 31, 473-483.

2. Calabrese EJ, Aulerich RJ, Padgett GA (1992): Mink as a predictive model in toxicology. Drug Metab Rev, 24, 559578.

3. Carlisle CH, Wu JX, Heath TJ (1995): Anatomy of the portal and hepatic veins of the dog: a basis for systematic evaluation of the liver by ultrasonography. Vet Radiol Ultrasoun, 36, 227-233.

4. Evans HE, de Lahunta A (2010): Guide to the Dissection of the Dog. Elsevier Saunders, St. Louis.

5. Gupta SC, Gupta CD, Arora AK (1977): Intrahepatic branching patterns of portal vein. A study by corrosion cast. Gastroenterology, 72, 621-624.

6. Hadžiomerović N, Avdić R, Tandir F, et al (2016): Intrahepatic distribution of the hepatic veins and biliary ducts in American mink liver (Mustela vison). J Vet Anat, 9, 15-23.

7. Heath T (1968): Origin and distribution of portal blood in the sheep. Am J Anat, 122, 95-106.

8. Heath T, House B (1970): Origin and distribution of portal blood in the cat and rabbit. Am J Anat, 127, 71-80.

9. Jepsen JR, d'Amore F, Baandrup U, et al (2009): Aleutian mink disease virus and humans. Emerg Infect Dis, 15, 2040-2042.

10. Kalt DJ, Stump JE (1993): Gross anatomy of the canine portal vein. Anat Histol Embryol, 22, 191-197.

11. Katica M, Hadžiomerović N (2020): Činčila, gerbil $i$ kanadska lasica kao potencijalni pokusni modeli u biomedicini. Veterinaria, 69, 91-94. 
12. Kirkeby S, Hammer AS, Hoiby N, et al (2017): Experimental Pseudomonas aeruginosa mediated rhino sinusitis in mink. Int J Pediatr Otorhinolaryngol, 96, 156163.

13. Kollias GV, Fernandez-Moran J (2015): Mustelidae. 476491 In: Miller RE, Fowler ME (Ed). Fowler's Zoo and Wild Animal Medicine. Elsevier Saunders, St Louis.

14. NAV (2017): The International Committee on Veterinary Gross Anatomical Nomenclature. 6th ed. (Revised version). Published by the Editorial Committee Hannover (Germany), Columbia, MO (USA), Ghent (Belgium), Sapporo (Japan).

15. Nickel R, Schummer A, Seiferle E (1981): The Anatomy of the Domestic Animals, Vol. 3: The circulatory system, the skin, and the cutaneous organs of domestic mammals. Verlag Paul Parey, Berlin.

16. Official Journal B \& H (2020): No. 34/02. Of November 22. The Veterinary Law in Bosnia and Herzegovina. Ministry of Foreign Trade and Economic Relations Sarajevo [pdf file]. Available at http://www.vet.gov.ba/pdffiles/Zakon_O_Vetrinarstvu/Vet erinary\%20Law.pdf (Accessed on September, 2020).
17. Osman FA, Wally YR, El-Nady FA, et al (2008): Gross anatomical studies on the portal vein, hepatic artery and bile duct in the liver of the pig. J Vet Anat, 1, 59-72.

18. Ranjbar R, Ghadiri AL (2011): Observation of intrahepatic branching pattern of the portal vein in water buffalo of Iran. Asian J Anim Vet Adv, 6, 508-516.

19. Singh B (2018): Dyce, Sack, and Wensing's textbook of Veterinary Anatomy. Elsevier, St. Louis.

20. Sleight DR, Thomford NR (1970): Gross anatomy of the blood supply and biliary drainage of the canine liver. Anat Rec, 166, 153-60.

21. Smith DG, Schenk MP (2000). Dissection Guide and Atlas to the Mink. Morton Publishing Company, Colorado.

22. Tadjalli M, Akhavan R (2003): Anatomical study on intrahepatic branches of portal vein in one humped camel (Camelus dromedarius). J Camel Pract Res, 10, 201-206.

23. Tadjalli M, Moslemy HR (2007): Intrahepatic ramification of the portal vein in the horse. Iran J Veterinary Re, 8, 116-122.

24. Uršič M, Ravnik D, Hribernik M, et al (2007): Gross anatomy of the portal vein and hepatic artery ramifications in dogs: Corrosion cast study. Anat Histol Embryol, 36, 8387. 